\title{
Polar optical phonon scattering and negative Krömer-Esaki- Tsu differential conductivity in bulk GaN
}

\author{
C. Bulutay, B.K. Ridley*, N.A. Zakhleniuk ${ }^{1}$ \\ Department of Electronic and Systems Engineering, University of Essex, Wivenhoe Park, Colchester, CO4 3SQ, UK
}

\begin{abstract}
$\mathrm{GaN}$ is being considered as a viable alternative semiconductor for high-power solid-state electronics. This creates a demand for the characterization of the main scattering channel at high electric fields. The dominant scattering mechanism for carriers reaching high energies under the influence of very high electric fields is the polar optical phonon (POP) emission. To highlight the directional variations, we compute POP emission rates along high-symmetry directions for the zinc-blende and wurtzite crystal phases of GaN. Our treatment relies on the empirical pseudopotential energies and wave functions. The scattering rates are efficiently computed using the Lehmann-Taut Brillouin zone integration technique. For both crystal phases, we also consider the negative differential conductivity possibilities associated with the negative effective mass part of the band structure. (C) 2001 Elsevier Science B.V. All rights reserved.
\end{abstract}

PACS: 72.10.Di; 72.20.Ht; 71.38. +i; 71.20.Nr

Keywords: A1. Computer simulation; B1. Nitrides; B2. Semiconducting III-V materials; B3. High electron mobility transistors

\section{Introduction}

The material parameters of $\mathrm{GaN}$ are indicative of very high-field transport regimes on the order of $\mathrm{MV} / \mathrm{cm}$, making it an attractive candidate for the high-power electronics applications. The high-field device operation requires a detailed knowledge of the electron transport characteristics such as energy-momentum relation and scattering mechanisms over the whole Brillouin zone (BZ). In the case of polar III-V compound materials, the

\footnotetext{
*Corresponding author. Tel.: +44-1206-872-873; fax:+441206-872-900.

E-mail address: bkr@essex.ac.uk (B.K. Ridley).

${ }^{1}$ Present address: Caswell Technology, Marconi Caswell, Towchester, Northants, NN12 8EQ,UK.
}

most effective scattering mechanism is due to the interaction with the lattice vibrations. This interaction is particularly strong in III-nitrides due to their outstanding ionicity [1]. It is about an order of magnitude stronger than in conventional III-V materials, like GaAs. Another important property of the nitrides is their large polar optical phonon (POP) energy. In GaN this is about 2.5 times higher than in GaAs. High POP energy creates an efficient channel for the electron energy loss. At such high electron energies, $\sim 1 \mathrm{eV}$, there is no other competitor to the POP scattering which would have the same magnitude of the rate of the momentum and the energy relaxation.

A realistic high-field analysis of the electronic device performance inevitably invokes a full-band treatment as the carriers under the influence of 
such fields move further away from the band edge where their scattering rate depends on the details of the band structure. In this paper, we consider both the zinc-blende (ZB) and wurtzite (WZ) band structures of $\mathrm{GaN}$ and present a full-band analysis of the POP scattering of the conduction band $(\mathrm{CB})$ electrons within the empirical pseudopotential band structure framework $[2,3]$.

\section{Theoretical approach}

The scattering rate due to POP emission based on Fermi's golden rule, considering only normalprocesses is given by

$$
\begin{aligned}
W_{j, m}(\vec{k})= & \sum_{m^{\prime}} \int \mathrm{d}^{3} k^{\prime} \hat{W}_{j, m^{\prime}}\left(\vec{k}^{\prime}\right) \delta\left(E_{m^{\prime}}\left(\vec{k}^{\prime}\right)\right. \\
& \left.-E_{m}(\vec{k})+\hbar \omega_{j}(\vec{q})\right) \\
= & \sum_{m^{\prime}} \int_{S} \mathrm{~d} S \frac{\hat{W}_{j, m^{\prime}}\left(\vec{k}^{\prime}\right)}{\left|\nabla E_{m^{\prime}}\left(\vec{k}^{\prime}\right)\right|}
\end{aligned}
$$

where

$$
\begin{aligned}
\hat{W}_{j, m^{\prime}}\left(\vec{k}^{\prime}\right)= & \frac{2 \pi}{\hbar} \frac{V}{(2 \pi)^{3}} \Delta_{m^{\prime}, m}\left(\vec{k}^{\prime}, \vec{k}\right) \\
& \times\left|C_{j}(\vec{q})\right|^{2}\left[n_{j}(\vec{q})+1\right] .
\end{aligned}
$$

Labels $m, \vec{k}$ represent the initial-state electron band index and wave vector, respectively; the primed indices correspond to the final-state, after the scattering event. The cell-periodic overlap parameter is given by $\Delta_{m^{\prime}, m}\left(\vec{k}^{\prime}, \vec{k}\right)=$ $\left|(1 / \Omega) \int_{\Omega} u_{m^{\prime}, \overrightarrow{k^{\prime}}}^{*}(\vec{r}) u_{m, \vec{k}}(\vec{r}) \mathrm{d}^{3} r\right|^{2}$, where $u_{m, \vec{k}}(\vec{r})$ is the

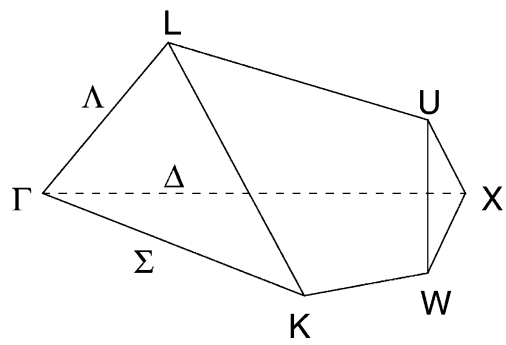

(a) cell-periodic part of the Bloch function, and $\Omega$ is the volume of the primitive cell. The integration is over the surface $S$, described by the energy conservation relation of one-phonon-emission process, $E_{m^{\prime}}\left(\vec{k}^{\prime}\right)=E_{m}(\vec{k})-\hbar \omega_{j}(\vec{q})$. Here $\hbar \omega_{j}(\vec{q})$ is the phonon energy corresponding to wave vector $\vec{q}=\vec{k}^{\prime}-\vec{k}$ mapped to first $\mathrm{BZ}$, and $j$ is the polar optical phonon branch index. For WZ structure the polar phonon branches are longitudinaloptical (LO)- and transverse-optical (TO)-like [4,5], whereas in the ZB case it is a pure LO mode. The associated electron-POP coupling coefficients are given by [5]

$$
\begin{aligned}
\left|C_{\text {LO_like }}(\vec{q})\right|^{2} \cong & \frac{2 \pi \mathrm{e}^{2} \hbar}{V q^{2} \Omega_{\mathrm{L}}\left(\theta_{\vec{q}}\right)}\left[\frac{\sin ^{2}\left(\theta_{\vec{q}}\right)}{\left(\frac{1}{\varepsilon_{\perp}^{\infty}}-\frac{1}{\varepsilon_{\perp}^{0}}\right) \omega_{\perp \mathrm{L}}^{2}}\right. \\
& \left.+\frac{\cos ^{2}\left(\theta_{\vec{q}}\right)}{\left(\frac{1}{\varepsilon_{z}^{\infty}}-\frac{1}{\varepsilon_{z}^{0}}\right) \omega_{z \mathrm{~L}}^{2}}\right]^{-1}
\end{aligned}
$$

$$
\begin{aligned}
& \left|C_{\mathrm{TO} \_ \text {like }}(\vec{q})\right|^{2}=\frac{2 \pi \mathrm{e}^{2} \hbar}{V q^{2} \gamma \Omega_{\mathrm{T}}\left(\theta_{\vec{q}}\right)} \\
& \times \frac{\left(\omega_{\perp}^{2}-\omega_{z}^{2}\right)^{2} \sin ^{2}\left(\theta_{\vec{q}}\right) \cos ^{2}\left(\theta_{\vec{q}}\right)}{\left(\varepsilon_{\perp}^{0}-\varepsilon_{\perp}^{\infty}\right) \omega_{\perp}^{2} \cos ^{2}\left(\theta_{\vec{q}}\right)+\left(\varepsilon_{z}^{0}-\varepsilon_{z}^{\infty}\right) \omega_{z}^{2} \sin ^{2}\left(\theta_{\vec{q}}\right)}, \\
& \left|C_{\mathrm{LO}}(\vec{q})\right|^{2}=\frac{2 \pi \mathrm{e}^{2} \hbar \omega_{\mathrm{LO}}}{V q^{2}}\left(\frac{1}{\varepsilon^{\infty}}-\frac{1}{\varepsilon^{0}}\right),
\end{aligned}
$$

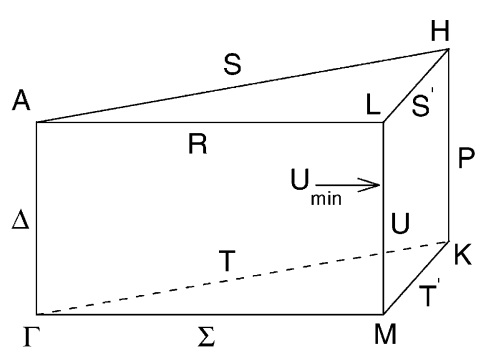

(b)

Fig. 1. Schematics of the irreducible wedges of ZB (a) and WZ (b) structures, indicating the high-symmetry points and lines. $\mathrm{U}_{\text {min }}$ point of the $\mathrm{WZ}$ phase is located on the $\mathrm{M}-\mathrm{L}$ line at two-thirds a distance away from the M point. 
where $V$ is the total crystal volume which cancels in the final expression, $\theta_{\vec{q}}$ is the angle phonon wave vector $\vec{q}$ makes with the crystal $c$-axis, $\omega_{\perp}\left(\omega_{z}\right)$ is the TO phonon frequency perpendicular to (along) the $c$-axis, $\omega_{\perp \mathrm{L}}\left(\omega_{z \mathrm{~L}}\right)$ is the LO phonon frequency perpendicular to (along) the $c$-axis, $\varepsilon_{\perp}^{\infty}\left(\varepsilon_{z}^{\infty}\right)$ is the high frequency dielectric constant perpendicular to (along) the $c$-axis. The use of Lyddane-Sachs-Teller relation gives the static dielectric constants as: $\varepsilon_{\perp}^{0}=\varepsilon_{\perp}^{\infty} \omega_{\perp \mathrm{L}}^{2} / \omega_{\perp}^{2}$ and $\varepsilon_{z}^{0}=\varepsilon_{z}^{\infty} \omega_{z \mathrm{~L}}^{2} / \omega_{z}^{2}$. The first two of the expressions in Eq. (2) are for the WZ structure while the last expression is for the $\mathrm{ZB}$ structure. The angledependent phonon frequencies in the $\mathrm{WZ}$ case are given by $[4,5]$

$$
\begin{gathered}
\Omega_{\mathrm{L}}\left(\theta_{\vec{q}}\right)=\sqrt{\omega_{z \mathrm{~L}}^{2} \cos ^{2} \theta_{\vec{q}}+\omega_{\perp \mathrm{L}}^{2} \sin ^{2} \theta_{\vec{q}}}, \\
\Omega_{\mathrm{T}}\left(\theta_{\vec{q}}\right)=\sqrt{\omega_{z}^{2} \sin ^{2} \theta_{\vec{q}}+\omega_{\perp}^{2} \cos ^{2} \theta_{\vec{q}}} .
\end{gathered}
$$

The $\mathrm{ZB}$ case is recovered by setting $\omega_{\perp}=\omega_{z} \equiv$ $\omega_{\mathrm{TO}}$ and $\omega_{\perp \mathrm{L}}=\omega_{z \mathrm{~L}} \equiv \omega_{\mathrm{LO}}$ in the above equations. We use the following data [6] for the WZ GaN phonons: $\hbar \omega_{z}=66.08 \mathrm{meV}$, $\hbar \omega_{z \mathrm{~L}}=91.13 \mathrm{meV}, \quad \hbar \omega_{\perp}=69.55 \mathrm{meV}, \quad \hbar \omega_{\perp \mathrm{L}}=$ $92.12 \mathrm{meV}$, and $\varepsilon_{\perp}^{\infty}=\varepsilon_{z}^{\infty}=5.29$. To be consistent with this data we use for $\mathrm{ZB}$ phase $\mathrm{GaN}$ phonons: $\hbar \omega_{\mathrm{LO}}=92.12 \mathrm{meV}$ and $\varepsilon^{0}=9.28$ and $\varepsilon^{\infty}=5.29$. Note that optical phonon energy is more than 3.5 times the thermal energy $(\sim 25 \mathrm{meV})$ at room temperature. For this reason, phonon occupation probability, $n_{j}(\vec{q})$, will be negligible up to room temperature, so that we can essentially set $n_{j}(\vec{q}) \equiv 0$. With the same reasoning we ignore the POP absorption processes in the electron scattering.

The Lehmann-Taut BZ integration technique [7] is very suitable for the computation of the scattering rate expression shown in Eq. (1). Accordingly, we divide the irreducible wedge (IW) (cf. Fig. 1) of the first BZ into fine tetrahedra, and store the band energies and the cell-periodic overlap parameters at the nodes of these tetrahedra. Even though the term $\hat{W}_{j, m^{\prime}}\left(\vec{k}^{\prime}\right)$ is not confined to the IW, ZB and WZ point-group symmetry operations [8,9] can be applied to generate the images of the points in IW, and hence, the overall integration can essentially be performed exploiting the tetrahedra in IW. It needs to be mentioned that very fine division of the IW ( $\sim 400,000$ tetrahedra) is necessary to reduce the computational ripple, especially favoring the high-symmetry points where satellite valleys are present. Still, this approach is much more timeefficient than the direct integration of the scattering rate [2].
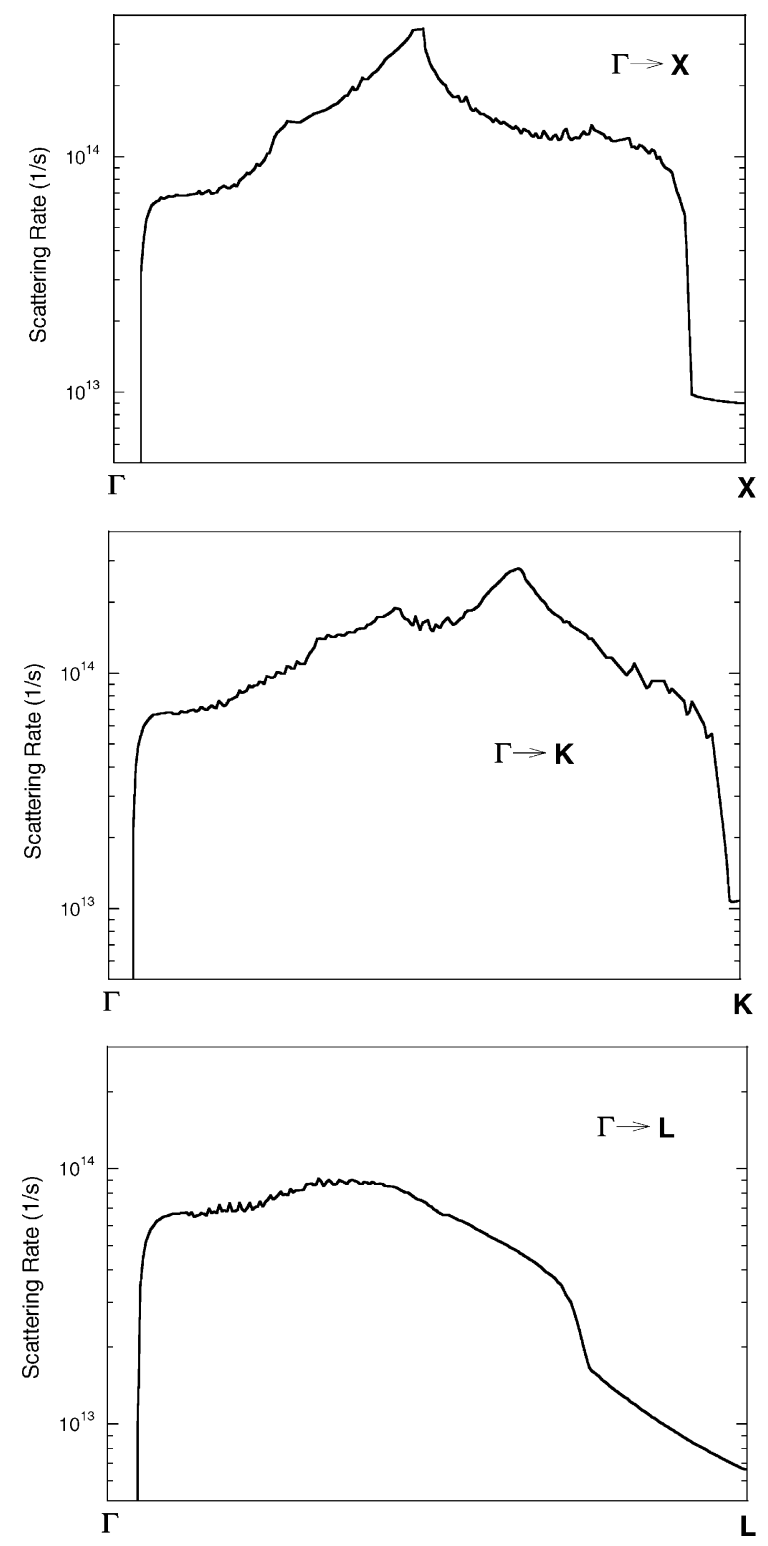

Fig. 2. POP scattering rates of $\mathrm{CB}$ electrons for $\mathrm{ZB} \mathrm{GaN}$ along the directions $\Gamma-\mathrm{X}, \Gamma-\mathrm{K}$, and $\Gamma-\mathrm{L}$. 


\section{Results}

Starting from the conduction band (CB) minimum at the $\Gamma$ point, we trace the POP scattering rates of the $\mathrm{CB}$ electrons, assuming a very high electric field applied along a high-symmetry line: $\Gamma-\mathrm{X}, \Gamma-\mathrm{K}$, and $\Gamma-\mathrm{L}$ for $\mathrm{ZB}$, and $\Gamma-\mathrm{M}, \Gamma-\mathrm{K}, \Gamma-\mathrm{A}$ and $\Gamma-\mathrm{U}_{\min }$ directions for $\mathrm{WZ}$ phases, see Fig. 1 for the directions. Due to $\mathrm{BZ}$ folding along $\Gamma-\mathrm{A}$ direction in $\mathrm{WZ}$ case [2], we actually follow an extended trajectory as $\Gamma_{1}-\mathrm{A}-\Gamma_{3}$. The results are shown in Figs. 2 and 3 for both crystal phases. Notably, there is a significant deviation of the scattering rate from its value near the $\mathrm{CB}$ minimum, especially when the scattering to other satellite valleys is energetically possible. The TOlike mode's scattering is seen to be more than two orders of magnitude weaker than the LO-like mode. For both crystal phases satellite valleys play major role in the quantitative value of the scattering rates. $\Gamma-\mathrm{L}$ direction in $\mathrm{ZB}$ has the lowest scattering rate which is due to the absence of an intravalley POP emission along this direction [10].

Using these scattering rates, we can estimate the high-field drift velocity behavior based on Esaki and Tsu's phenomenological semiclassical treatment [11], where the average drift velocity is given by

$v_{\mathrm{d}}=\mathrm{e} F \hbar^{-2} \int_{0}^{\infty} E^{\prime \prime} \mathrm{e}^{-t / \tau} \mathrm{d} t$

where $E^{\prime \prime}$ is the curvature of the energy band diagram along the applied field's direction $\vec{F}$, sampled at the point $\vec{k}(t)=\mathrm{e} \vec{F} t / \hbar$, for an electron originating from the $\Gamma$ point. For the scattering
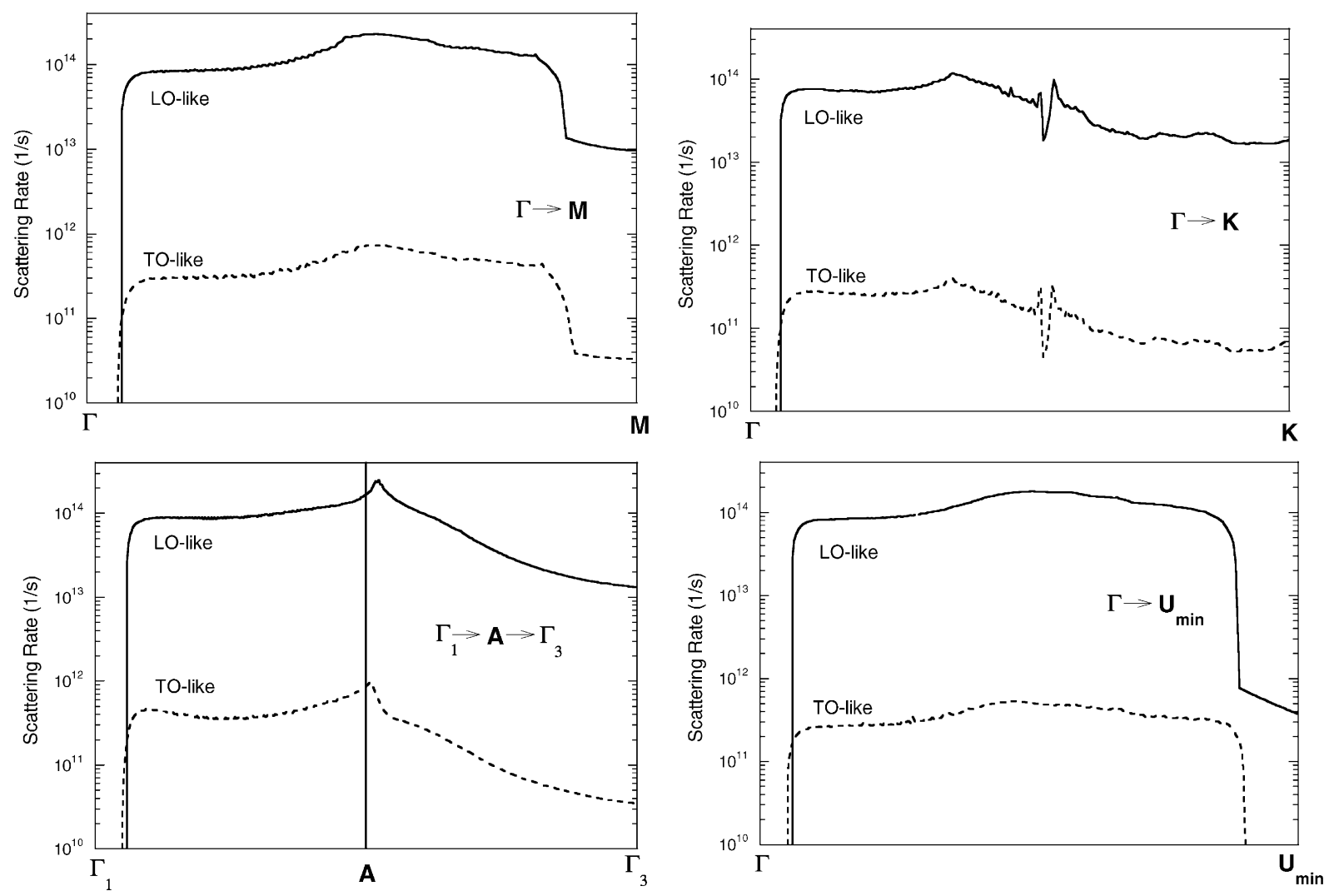

Fig. 3. POP scattering rates of $\mathrm{CB}$ electrons for $\mathrm{WZ} \mathrm{GaN}$ along the directions $\Gamma-\mathrm{M}, \Gamma-\mathrm{K}, \Gamma-\mathrm{A}$ and $\Gamma-\mathrm{U}_{\min }$. Due to $\mathrm{BZ}$ folding along $\Gamma-\mathrm{A}$ direction an extended trajectory, $\Gamma_{1}-\mathrm{A}-\Gamma_{3}$, is followed. 


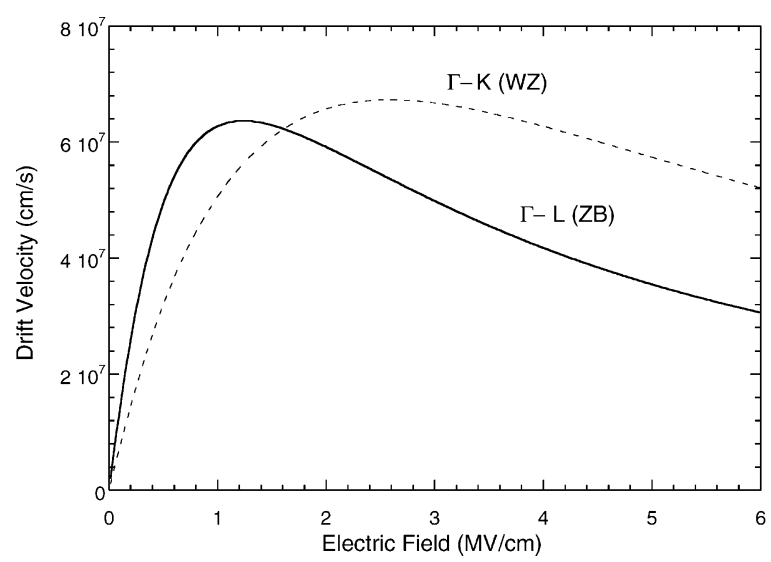

Fig. 4. High-field-related drift velocity versus electric field behavior along $\Gamma-\mathrm{L}(\mathrm{ZB})$ and $\Gamma-\mathrm{K}(\mathrm{WZ})$ directions.

time $\tau$ in this equation, we use the value given by the maximum scattering rate in each direction, monitoring the full period of the electron's trajectory in the wave vector-space (for certain directions, exceeding the first BZ boundary). We would like to stress that such an approach makes sense only at very high electric fields, say above $0.5 \mathrm{MV} / \mathrm{cm}$. With this caution, we provide in Fig. 4 the corresponding drift velocity behavior along $\Gamma-\mathrm{L}(\mathrm{ZB})$ and $\Gamma-\mathrm{K} \quad(\mathrm{WZ})$ directions. Electrons reaching the negative effective mass part of the band structure before undergoing a scattering event, give rise to a negative differential conductivity (NDC). As this idea was initially conjectured by Krömer [12] and later applied to superlattices by Esaki and Tsu [11], it can be distinguished from other NDC mechanisms [13] by Krömer-Esaki-Tsu NDC. The effect needs quite high electric fields, with the lowest onset field being $\sim 1 \mathrm{MV} / \mathrm{cm}$ along $\Gamma-\mathrm{L}$ direction of the $\mathrm{ZB}$ phase. As a follow-up a more refined analysis will be appropriate to verify this phenomenological estimation.

\section{Conclusions}

We present a full-band POP scattering analysis of $\mathrm{CB}$ electrons for the $\mathrm{ZB}$ and $\mathrm{WZ}$ crystal phases of $\mathrm{GaN}$. There is a significant deviation of the scattering rate from its value near the $\mathrm{CB}$ edge when the scattering to other satellite valleys becomes energetically possible. $\Gamma-\mathrm{L}$ direction of $\mathrm{ZB} \mathrm{GaN}$ has the lowest scattering rate characterized by a scattering time of $11 \mathrm{fs}$. Especially for this direction the observation of Krömer-Esaki-Tsu NDC seems possible beyond a field of roughly $1 \mathrm{MV} / \mathrm{cm}$.

\section{Acknowledgements}

This work is supported by ONR (N00014-99-10014) and $\operatorname{EPSRC}(\mathrm{GR} / \mathrm{L} / 56725)$.

\section{References}

[1] A. García, M. L. Cohen, Phys. Rev. B 47 (1993) 4215 and 4221.

[2] C. Bulutay, B.K. Ridley, and N.A. Zakhleniuk, Phys. Rev. B 62 (2000) 15754.

[3] R. Wang, P.P. Ruden, J. Kolnik, I. Oguzman, K.F. Brennan, J. Phys. Chem. Solids 58 (1997) 913.

[4] R. Loudon, Adv. Phys. 13 (1964) 423.

[5] B.C. Lee, K.W. Kim, M. Dutta, M.A. Stroscio, Phys. Rev. B 56 (1997) 997.

[6] T. Azuhata, T. Sota, K. Suzuki, S. Nakamura, J. Phys. Condens. Matter 7 (1995) L129.

[7] G. Lehmann, M. Taut, Phys. Stat. Sol. B 54 (1972) 469.

[8] N. Sano, A. Yoshii, Phys. Rev. B 45 (1992) 4171.

[9] I.H. Oguzman, J. Kolník, K.F. Brennan, R. Wang, T.N. Fang, P.P. Ruden, J. Appl. Phys. 80 (1996) 4429.

[10] C. Bulutay, B.K. Ridley, and N.A. Zakhleniuk, Appl. Phys. Lett. 77 (2000) 2707.

[11] L. Esaki, R. Tsu, IBM J. Res. Dev. 14 (1970) 61.

[12] H. Krömer, Phys. Rev. 109 (1958) 1856.

[13] N. Balkan (Ed.), Hot Electrons in Semiconductors, Oxford University Press, Oxford, 1998. 